\title{
Parameter degeneracies and new plots in neutrino oscillations
}

\author{
Osamu Yasuda \\ Department of Physics, Tokyo Metropolitan University \\ 1-1 Minami-Osawa Hachioji, Tokyo 192-0397, Japan
}

\begin{abstract}
It is shown that plots of constant probabilities in the $\left(\sin ^{2} 2 \theta_{13}, 1 / s_{23}^{2}\right)$ plane enable us to see eightfold degeneracy easily. Using this plot, I discuss how an additional long baseline measurement resolves degeneracies after the JPARC experiment measures the oscillation probabilities $P\left(\nu_{\mu} \rightarrow \nu_{e}\right)$ and $P\left(\bar{\nu}_{\mu} \rightarrow \bar{\nu}_{e}\right)$ at $\left|\Delta m_{31}^{2}\right| L / 4 E=\pi / 2$.
\end{abstract}

It is known that even if the values of the oscillation probabilities $P\left(\nu_{\mu} \rightarrow \nu_{e}\right)$ and $P\left(\bar{\nu}_{\mu} \rightarrow \bar{\nu}_{e}\right)$ are exactly given, we cannot determine uniquely the values of the oscillation parameters due to eightfold parameter degeneracy. To see how the eightfold degeneracy is lifted, it is necessary for the plot to give eight different points for different eight solutions. In Ref. 1 it was shown that the solution given by $P\left(\nu_{\mu} \rightarrow \nu_{e}\right)=$ const. and $P\left(\bar{\nu}_{\mu} \rightarrow \bar{\nu}_{e}\right)=$ const. gives a hyperbola in the $\left(\sin ^{2} 2 \theta_{13}, 1 / s_{23}^{2}\right)$ plane in most cases, as is shown in Fig 1(a), so that this plot is useful to see how the eightfold degeneracy is resolved.

It is expected that the JPARC experiment will measure the oscillation probabilities $P\left(\nu_{\mu} \rightarrow \nu_{e}\right)$ and $P\left(\bar{\nu}_{\mu} \rightarrow \bar{\nu}_{e}\right)$ at $\left|\Delta m_{31}^{2}\right| L / 4 E=\pi / 2$. Here I would like to discuss how the eightfold degeneracy is resolved by an additional experiment. From the JPARC experiment on $\nu_{\mu} \rightarrow \nu_{e}$ and $\bar{\nu}_{\mu} \rightarrow \bar{\nu}_{e}$ at $\left|\Delta m_{31}^{2}\right| L / 4 E=\pi / 2$, we can deduce the value of the $\mathrm{CP}$ phase $\delta$ up to the eightfold ambiguity. The trajectories given by the JPARC experiment at $\left|\Delta m_{31}^{2}\right| L / 4 E=\pi / 2$ turn out to be straight lines. For each value of $\delta$, from the third experiment on $\nu_{\mu} \rightarrow \nu_{e}$ (or $\bar{\nu}_{\mu} \rightarrow \bar{\nu}_{e}$ ) one obtains a unique trajectory in the $\left(\sin ^{2} 2 \theta_{13}, 1 / s_{23}^{2}\right)$ plane. Putting the trajectories of the third and JPARC experiments together in the $\left(\sin ^{2} 2 \theta_{13}, 1 / s_{23}^{2}\right)$ plane, we find that (i) in general it is difficult to resolve the $\theta_{23}$ ambiguity as is shown in Fig प(b) (this is not the case with the silver channel $\nu_{e} \rightarrow \nu_{\tau}$ ), (ii) the third experiment with longer baseline and lower energy re-
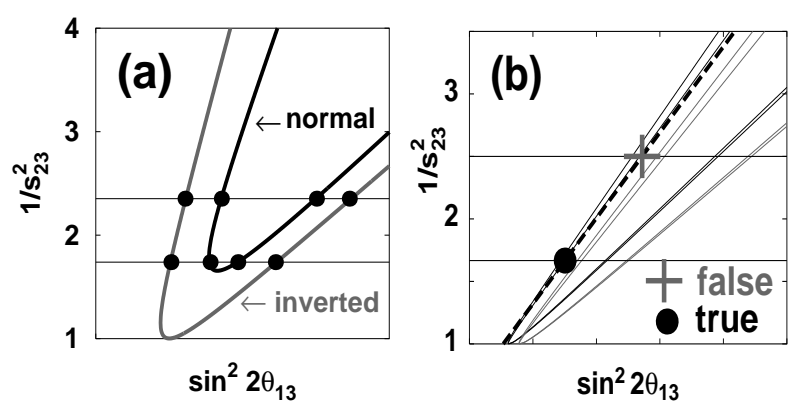

Figure 1. (a) Trajectories of solutions given by $P\left(\nu_{\mu} \rightarrow \nu_{e}\right)=$ const. and $P\left(\bar{\nu}_{\mu} \rightarrow \bar{\nu}_{e}\right)=$ const. and the eightfold degeneracy. (b) Trajectories given by the third experiment on $\nu_{\mu} \rightarrow \nu_{e}$ (solid lines) and the JPARC experiment (dashed line), where black (gray) lines are for normal (inverted) hierarchy, and the blob (cross) stands for the true (false) solution.

solves the $\operatorname{sgn}\left(\Delta m_{32}^{2}\right)$ ambiguity better, and (iii) the $\delta \leftrightarrow \pi-\delta$ ambiguity is resolved better for $\pi / 2<\left|\Delta m_{31}^{2}\right| L / 4 E<\pi$.

This work was supported in part by Grants-inAid for Scientific Research No. 16540260 and No. 16340078, Japan Ministry of Education, Culture, Sports, Science, and Technology.

\section{REFERENCES}

1. O. Yasuda, New J. Phys. 6 (2004) 83 arXiv:hep-ph/0405005. 